\title{
Fluorescence in cola drinks and caramel
}

\section{Carlos Eugenio Tapia Ayuga}

Departamento de Astrofísica, Universidad Complutense, Madrid, Spain.

\section{Alfredo Luis}

Departamento de Óptica, Facultad de Ciencias Físicas, Universidad Complutense, Madrid, Spain. E-mail: alluis@fis.ucm.es

THE PHYSICS TEACHER, Vol. 52, p. 576 JANUARY 2014.

When illuminated by a green laser pointer (532 nm wavelength) in a darkened room, the light path within popular cola drinks becomes reddish or even deep red, in sharp contrast with the green color in air, tube water and container walls (see Fig. 1). We find extremely formative the physical reasoning that, starting from laser monochromaticity and darkened environment, leads to frequency-changing fluorescence as the most plausible explanation. We have observed the effect in reddish cola drinks having in common just the caramel-based food coloring E150d, while the effect is absent in some other reddish drinks without such ingredient. This led us to try the effect in home-prepared caramel dissolved in water, obtaining a more pronounced effect for large concentrations (see Fig. 1).

The frequency-changing fluorescence hypothesis can be tested in several different ways, such as spectrum observation with a simple spectroscope. The most powerful contrast is provided by recording the fluorescence spectra by forming an image of the pointer beam within the liquids in the input of an spectrometer. The spectra are displayed in Fig. 2 being compared with the spectrum when the samples are removed. The red-shifted fluorescence is clearly visible confirming that the color change is not a visual deception. Note the stronger red shift in the most concentrated caramel sample, and the equality of the background noise for all spectra.

All measurements have been performed at the Laboratorio de Investigación Cientifica Avanzada (LICA-UCM) of the Moncloa Campus of International Excellence (UCM-UPM) with support from the Spanish Programa Nacional de Astronomía y Astrofísica under grant AYA2009-10368 and Consolider-Ingenio 2010 Program grant CSD2006-00070: First Science with the GTC from Spanish MICINN. We thank Dr. R. Weigand for a critical reading of the manuscript and valuable suggestions.

See also G. R. Gore, "Fun with fluorescence in olive oil," The Physics Teacher 50, 377378 (September 2012) and M. F. Wahab, G. R. Gore "Deeper insight into fluorescenceexcitation of molecules by light," The Physics Teacher 51, 306-308 (May 2013) 


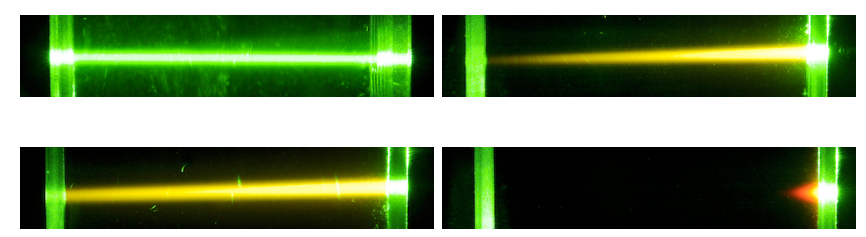

Figure 1.- Green laser pointer in tube water (upper left), cola drink (upper right), less concentrated caramel (lower left), and more concentrated caramel (lower right).

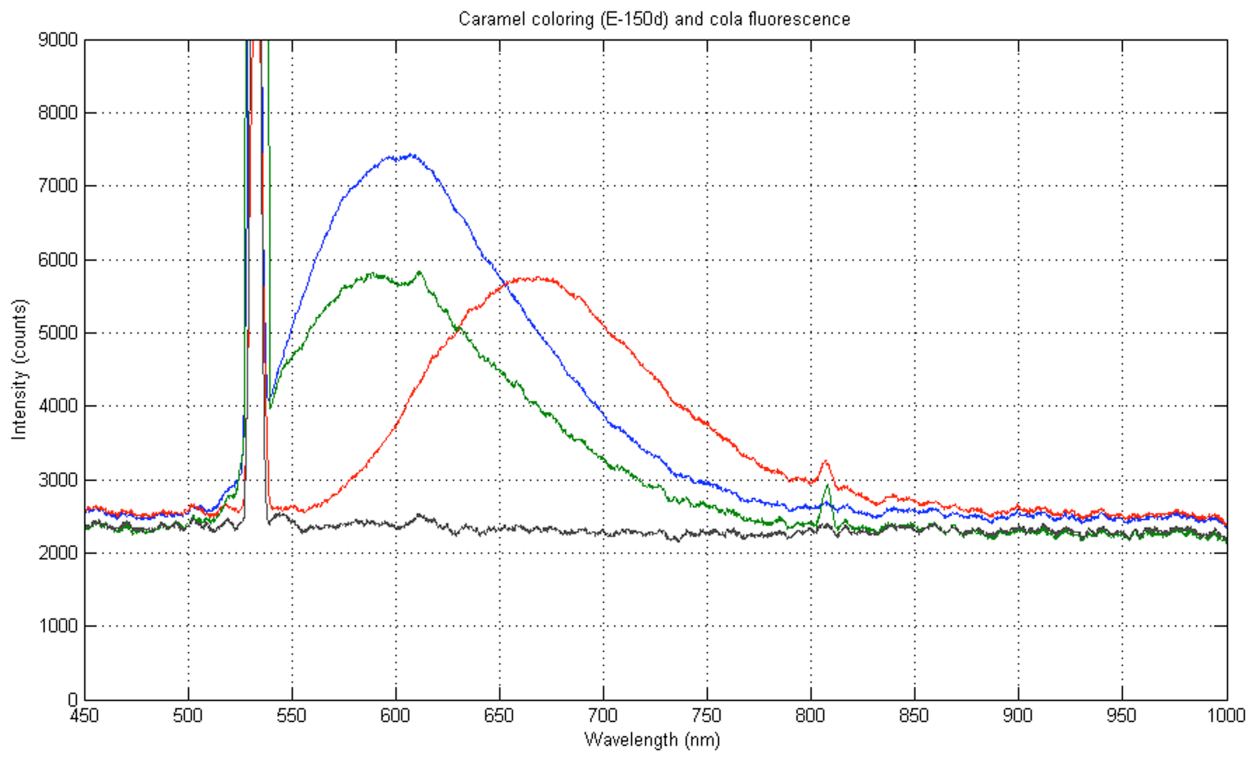

Figure 2.- Recorded spectra of the pointer light within cola drink (green line) less concentrated caramel (blue line), more concentrated caramel (red line), and in air (grey line). 